\title{
The Effect of Decreased Catecholamine Transmission on ERP Indices of Selective Attention
}

\author{
Anne-Marie Shelley, Ph.D., Stanley V. Catts, M.D., Philip B. Ward, Ph.D., Sally Andrews, Ph.D.,
} Penny Mitchell, B.Sc., Patricia Michie, Ph.D., and Nathaniel McConaghy, D.Sc.

This study examines the effect of decreased catecholamine transmission on event-related potential (ERP) indices of selective attention. Intravenous clonidine $(1.5 \mu \mathrm{g} / \mathrm{kg}$ Catapres), droperidol (15 $\mu \mathrm{g} / \mathrm{kg}$ Droleptan), or placebo were administered to healthy adult males prior to performance of a multidimensional auditory selective attention task (SAT) in which dichotically presented sequences of tone pips varied on dimensions of location (left or right ear), pitch (high or low), and duration (short or long). Subjects were required to make a button press response to infrequent "target" stimuli that matched a prespecified stimulus on the three dimensions. ERPS

KEY WORDS: Catecholamines; Clonidine; Droperidol; ERPS; Selective attention; Processing negativity; P3

Selective attention is the ability to attend to relevant information and ignore irrelevant information (Posner and Boies 1971). Converging lines of evidence have implicated catecholamines (CAs) in the neural substrate of selective attention (for reviews see Clark et al. 1987a, 1987b; Oades, 1985). Animal studies have demonstrated that decreases in noradrenaline (NA) (Mason 1980; Pineda et al. 1989) and either decreased dopamine (DA) (Oades 1981, 1982) or increased DA (Beninger 1983; Crider et al. 1982) are as-

From the School of Psychology (SA, PM) and School of Psychiatry (A-MS, SVC, PBW, NMCC), University of New South Wales, Psychiatry Department (SVC.), Prince of Wales Hospital, School of Behavioral Sciences (PM), Macquarie University.

Address correspondence to: Dr. Anne-Marie Shelley, Department of Psychiatry, Albert Einstein College of Medicine, 1300 Morris Park Ave., Bronx, NY, 10461.

Received October 2, 1995; revised July 16, 1996; accepted August 13,1996 were recorded during the task. Clonidine led to a significant increase of processing negativity (PN) over 200-400 ms at the irrelevant location. Droperidol led to a significant increase in reaction time $(R T)$, a significant decrease in hit rate, and an attenuation of PN over the 200- to 400-ms and 400- to 700-ms epochs. Neither substance led to a significant change in P3 amplitude. The role of catecholamines in the selective attention subprocesses of "tuning" and "switching" is discussed. (C) 1997 American College of Neuropsychopharmacology [Neuropsychopharmacology 16:202-210, 1997]

sociated with selective attention deficits. Pharmacological studies with normal human subjects have confirmed findings from animal studies and demonstrated that CA antagonists interfere with behavioral performance on attentional tasks (Clark et al. 1986a, 1986b). In addition, attentional abnormalities in several clinical disorders such as schizophrenia (Oades 1982a), attention deficit disorder (ADD) (Raskin et al. 1984), and Parkinson's disease (Hornykiewicz and Kish 1984) have been attributed to dysfunctional CA systems.

Despite these findings, the precise role of NA and DA in attentional subprocesses is yet to be fully elaborated. A number of researchers have proposed dichotomous frameworks, in which NA and DA activity are identified with specific attentional functions. For example, Tucker and Williamson (1984) hypothesized that NA modulates "arousal" by facilitating orientation and habituation mechanisms, whereas DA affects "activation" by expediting the selection and organization of motor acts. Clark et al. (1987a, 1987b) suggested that NA regu- 
lates "early" information or "stimulus" processing, whereas DA regulates "late" information or "response" processing. Oades (1985) proposed that NA is responsible for "tuning," or changes in the signal-to-noise ratio, whereas DA modulates "switching" between different sources of information. These frameworks are useful because they allow much of the available data to be summarized in simple terms, but their generality prevents them from providing an accurate, detailed analysis of $\mathrm{CA}$ involvement in the various substages of selective attention.

One factor that has constrained the development of a precise model of CA involvement in selective attention has been the reliance on behavioral indices, such as reaction time (RT) data. Behavioral measures have two inherent shortcomings. First, they lack the fine temporal resolution that is required to monitor real-time characteristics of task performance. Second, it is difficult to determine the extent to which unattended information is processed because overt behavioral responses are withheld for irrelevant stimuli (Bookbinder and Osman 1979). The use of event-related potentials (ERPs) to measure attentional processes eliminates these two problems. The time resolution of ERPS is in the millisecond range, so the latencies of different ERP components can provide fine-grained information about the temporal dynamics of the neural events involved in information processing (McCarley et al. 1991). Because ERPs measure electrophysiological brain events elicited by all sensory stimuli, regardless of relevance or whether overt behavioral responding is required, they enable estimation of the extent to which unattended, as well as attended, stimuli are processed (Näätänen 1990; Hansen and Hillyard 1983).

The multidimensional auditory selective attention task (SAT) (Hansen and Hillyard 1983) is an ERP paradigm that is specifically designed to examine the selectivity of attention. In the version of the SAT used in the present experiment subjects listened via headphones to sequences of tone pips. Stimuli varied along three dimensions: location (L) (left or right ear) was the "easy" attribute, pitch (P) was the "more difficult" discriminating factor, and duration (D) was the "most difficult" discrimination. Each of the four location-pitch combinations was equiprobable; within each location-pitch combination long duration tones were infrequent. Prior to each run, subjects were played the "target" (one of the four long duration tones) and instructed to make a button press response whenever they detected a stimulus that matches $(+)$ the prespecified target on all three dimensions $(\mathrm{L}+\mathrm{P}+\mathrm{D}+)$.

Short-duration (D) nontarget or "standard" stimuli elicit processing negativity ( $\mathrm{PN}$ ), which is thought to provide the most accurate index of the selectivity of attention (Näätänen et al. 1978; Näätänen and Michie 1979). "Early PN" (100-200 ms) is identified with the separation of ERPs at the attended location $(\mathrm{L}+)$ from ERPs at the unattended location $(\mathrm{L}-)$. For $\mathrm{L}-$ stimuli, early $\mathrm{PN}$ is followed by "later PN" (200-400 ms), which reflects the separation of the attended pitch $(\mathrm{L}+\mathrm{P}+)$ from the unattended pitch $(\mathrm{L}+\mathrm{P}-)$. Normally, there is no significant separation of ERPs on the basis of pitch at the unattended location. This ERP profile indicates that the selection of the more difficult pitch dimension is subsequent to and contingent on the easy location discrimination (Hansen and Hillyard 1983). This pattern is consistent with contingent or hierarchical models of dimension selection (Hawkins 1969; Snodgrass and Townsend 1980) but inconsistent with models that imply an independent, exhaustive analysis of dimensions and late selection (Deutsch and Deutsch 1963; Norman 1968). Thus "early" and "later" PN appear to reflect the selection of location and pitch. The time of separation allows the determination of the latency of the relevant attribute selection. A "very late $P^{\prime \prime}$ ( $400-700 \mathrm{~ms}$ ) has been proposed by Näätänen $(1982,1990)$ to index the maintenance and rehearsal of an "attentional trace" of the $\mathrm{L}+\mathrm{P}+\mathrm{D}+$ standard. ${ }^{1}$ The detection of targets in the SAT is indexed by the parietally maximal $\mathrm{P} 3$ component elicited by the $\mathrm{L}+\mathrm{P}+\mathrm{D}+$.

Näätänen's (1982) model of the functional significance of PN can be linked with Oades' (1985) concepts of "tuning" and "switching." Tuning, according to Oades, is the modulation of signal-to-noise ratio. Location and pitch PN, according to Näätänen, reflect enhanced processing of relevant stimulus dimensions. Presumably, PN can be viewed as a function of tuning. Tuning is affected by the manipulation of NA transmission, with decreased NA transmission causing reduced tuning and increased NA transmission causing enhanced tuning. Clonidine is an A2 adrenoceptor agonist commonly employed as an antihypertensive agent. By potentiating presynaptic inhibitory activity, clonidine inhibits the firing of noradrenergic neurons in the locus coeruleus, the nucleus of origin of NA pathways, and thereby decreases NA transmission to cortical and hippocampal areas (Svensson et al. 1975; Aghajanian 1982) Thus the administration of clonidine, a functional NA antagonist, was predicted to lead to decreased tuning, reflected in ERPs as a decrease in the amplitude of location- and pitch-related PN. However, previous studies that have examined the effects of clonidine on ERPs have found that it led to a reduction in the amplitude of the $\mathrm{P} 3$ component in the auditory modality (Duncan and Kaye 1986; Joseph and Sitaram 1989; Swick et al. 1988).

As the SAT employs multidimensional stimuli that are processed hierarchically, it is possible to relate Oades' (1985) concept of "switching" to the shifting of attention from the easy location discrimination to the more difficult

'Näätänen $(1982,1990)$ has proposed that it is the features of the standard most resembling the target (i.e., the $\mathrm{L}+\mathrm{P}+\mathrm{D}-$ ), rather than all the features of the target (i.e., the $\mathrm{L}+\mathrm{P}+\mathrm{D}+$ ) itself, that are assumed to be incorporated into the attentional trace, because the $\mathrm{L}+\mathrm{P}+\mathrm{D}-$ stimulus occurs three times as frequently as the $\mathrm{L}+\mathrm{P}+\mathrm{D}+$ stimulus and an attentional trace cannot be maintained for long without external reinforcement by the stimulus. 
pitch discrimination at $\mathrm{L}+$. Switching is affected by the manipulation of DA transmission, with increased DA transmission causing an increase in the likelihood of switching between alternative sources of information and decreased DA transmission causing a difficulty in initiating or switching between appropriate behavioral sequences. Droperidol is a short-acting neuroleptic agent of the butyrophenone group, that has potent DA receptor antagonist properties (Peroutka and Snyder 1980). Although droperidol also affects other monoamine systems and produces weak adrenergic blockade (Craig and Stitzel 1982), its chief effect is on the DA system. Thus the administration of droperidol, a DA antagonist, was predicted to lead to a deficit in switching from the relevant location to the relevant pitch attribute. This should be reflected in ERPs as a decrease in the amplitude of the pitch but not the location-related PN.

In addition to investigating pharmacological effects on ERPs, the present study also examined their effects on behavioral performance, in terms of "stimulus" and "response" processing. Previous research has shown that stimulus processing, reflected in hit rate and discriminating, is governed by both the NA and DA systems, whereas response processing, reflected in $\mathrm{RT}$, is governed by the DA system, (Clark et al. 1986a, 1986b, 1987b). Thus clonidine's specific inhibition of NA was predicted to disrupt stimulus processing, reflected in a decrease in hit rate and discrimination, but not response processing and therefore RT. Droperidol, chiefly a DA antagonist, was predicted to impair both stimulus and response processing, reflected in decreased hit rate and discrimination and increased RT, because of difficulties in motor initiation as a consequence of droperidol's pseudo-Parkinsonian side effects.

\section{MATERIALS AND METHODS}

\section{Subjects}

Ten normal right-handed males (age 20-32, mean 25.5 years) took part in all experimental sessions. Subjects had to pass a medical examination that included psychiatric and drug screening and provide informed consent prior to entry into the study.

\section{Design}

The clonidine (CLON), droperidol (DROP), and saline placebo (SAL) conditions reported in this paper were three conditions of a six-session repeated-measures study. A previous drug-free repeated-measures ERP study that employed the same multidimensional selective attention task as used in this experiment found that repetition of the task per se caused significant changes in ERP and behavioral indices (Shelley et al. 1991). The authors recommended that studies requiring repeated testing of subjects to investigate different treatment effects should aim to eliminate such repetition effects because they may confound interpretation of the effects of the variable under investigation. As ERP and behavioral changes of the largest magnitude occurred from Session 1 to Session 2 , it was recommended that repeated-measures designs include an initial recording session that is excluded from the evaluation of treatment effects. Although further ERP changes were observed over Session 2 to 6, they were of considerably smaller magnitude and could be expected to be counteracted by the use of counterbalancing or Latin Square designs. In addition, other studies have shown that initial testing can elicit more anxiety than later sessions, in which subjects feel more familiar with testing procedures (Lader 1980). This is particularly likely to occur in drug studies employing intravenous injections. Thus an initial placebo session that is excluded from statistical analyses should be employed to reduce "firstsession" effects, that is, ERP and behavioral changes due to the repetition of the task per se, and to familiarize subjects with the task and the drug administration procedure. Accordingly, SAL in the present study refers to the second of two placebo sessions, and the first saline session was excluded from all statistical analyses. The remaining two sessions employed opioid modulating agents and opioid data are reported elsewhere (Shelley et al. 1990). Recordings were spaced 1 week apart. The study was conducted according to a double-blind Latin Square design.

\section{Materials and Apparatus}

The task was adapted from Hansen and Hillyard (1983). Tones varying in location ("easy cue": left or right ear) and pitch ("difficult" cue: $1,047 \mathrm{~Hz}$ or $1,319 \mathrm{~Hz}$ ) were presented binaurally via headphones in random sequences (ISI: 200-500 ms). Within each equiprobable location-pitch combination, $72 \%$ of tones were of short (50 $\mathrm{ms}$ ) duration, and $28 \%$ were of long $(100 \mathrm{~ms})$ duration. Duration was used as target because duration information is not available at stimulus onset, making duration an unsuitable attribute for use in a channel. Subjects made a button-press response to long-duration tones of differing location-pitch combinations on each of eight runs. Target varied across runs. ERPs were created by sorting stimuli according to whether they matched or did not match the target on location $(\mathrm{L}+/ \mathrm{L}-)$, pitch $(\mathrm{P}+/ \mathrm{P}-)$, and duration $\left(\mathrm{D}+/ \mathrm{D}^{-}\right)$.

\section{Procedure}

EEG was recorded from 16 scalp electrodes, along with vertical and horizontal electrooculogram (EOG), with a bandpass of 0.01 to $30 \mathrm{~Hz}$. All electrodes were referenced to the nose. Electrophysiological data were sampled at 5 -ms intervals beginning $250 \mathrm{~ms}$ prestimulus and ex- 
tending 1,030 ms poststimulus. EEG epochs-accompanied eye movement artefact were excluded from analysis. Sweeps contaminated by incorrect behavioral responses (misses or false alarms) also were excluded because it is unknown whether subjects are on task. Subjects received either SAL, CLON $(1.5 \mu \mathrm{g} / \mathrm{kg})$, or DROP $(15 \mu \mathrm{g} / \mathrm{kg})$ IV approximately 10 minutes prior to a 40 -minute recording session under double-blind conditions. Systolic blood pressure (SBP), diastolic blood pressure (DBP), and heart rate (HR) were recorded pre-and postinjection and half-way through each session. A 12-item mood questionnaire (Shelley 1991) was administered immediately postinjection and half-way through each session.

\section{Data Analysis}

Behavioral performance data were analyzed for hit rate, false alarm rate, and reaction time (RT). Cardiovascular indices (SBP, DBP, and HR) were converted to change scores from pre- to postinjection. Mood scores were converted to composite scores on five dimensions: anxiety, affect, attention, alertness, and comfort. All behavioral indices were analyzed with two separate analyses of variance (ANOVAs), that compared the CLON and the DROP condition with the SAL condition, respectively. Previous research has indicated that $P N$ is maximal at $\mathrm{Fz}$, whereas $\mathrm{P} 3$ is maximal at $\mathrm{Pz}$ (Hansen and Hillyard 1983). Accordingly, all ERP measures were derived from $\mathrm{Fz}$, except for P3 measures, which were derived from Pz. D-ERPs were analyzed for mean amplitude over 100- to 200-ms, 200- to 400-ms, and 400- to 700-ms runs. The stimulus type was examined for the effect of location (the two $\mathrm{L}+$ standard ERPs compared to the two L- standard ERPs) and the effect of pitch (P+ standards vs. P - standards) and was first analyzed with two separate ANOVAs that compared the CLON and the DROP condition with the SAL condition, respectively.

Table 1. Mood, Cardiovascular, and Behavioral Performance Scores Following Clonidine, Droperidol, and Saline Administration

\begin{tabular}{lccc}
\hline & Clonidine & Droperidol & Saline \\
\hline Anxiety & $1.1(0.7)$ & $2.9(2.6)$ & $1.1(1.0)$ \\
Affect & $1.1(0.9)$ & $2.9(2.1)$ & $1.0(1.3)$ \\
Comfort & $1.4(1.0)$ & $2.6(2.1)$ & $1.4(1.2)$ \\
Altertness & $3.6(2.7)$ & $3.6(2.3)$ & $1.9(1.9)$ \\
Attention & $4.3(3.1)$ & $5.0(3.7)$ & $2.1(2.6)$ \\
Systolic blood pressure & $11.9(12.7)$ & $6.9(9.9)$ & $6.7(8.1)$ \\
Diastolic blood pressure & $4.2(6.0)$ & $5.2(7.3)$ & $7.3(7.0)$ \\
Heart rate & $5.4(10.8)$ & $2.7(10.6)$ & $6.8(8.2)$ \\
Hit rate $(\%)$ & $71.0(10.5)$ & $63.5(15.5)$ & $76.1(12.1)$ \\
False alarm rate $(\%)$ & $5.1(4.5)$ & $3.8(3.7)$ & $3.6(3.1)$ \\
Reaction time & $529.5(26.5)$ & $556.4(85.8)$ & $499.3(51.9)$
\end{tabular}

Mood and behavioral data are expressed as means (SD in parentheses), cardiovascular data as change scores from pre- to postinjection.
To evaluate the hierarchical nature of processing over the 200- to 400-ms and 400- to 700-ms tests, mean amplitude of $\mathrm{P}+$ and $\mathrm{P}-\mathrm{ERPs}$ were compared at the attended and the unattended location using follow-up $t$-tests that assessed the relative processing of pitch. D+ ERPs were analyzed for $\mathrm{P} 3$ mean amplitude, peak amplitude, and latency over 300 to $800 \mathrm{~ms}$ at $\mathrm{Pz}$ in the $\mathrm{L}+\mathrm{P}+\mathrm{D}+\mathrm{ERP}$, using separate ANOVAs that compared the CLON and the DROP condition with the SAL condition, respectively. $\mathrm{L}+\mathrm{P}+\mathrm{D}+$ data are presented only for $\mathrm{P} 3$ analysis because $\mathrm{P} 3$ obscures processing negativity.

\section{RESULTS}

\section{Behavioral Data}

Mood Scales. Self-ratings of mood indicated that following CLON subjects felt significantly less alert $[F(1,9)=$ 9.97, $p<.012]$ and less attentive $[F(1,9)=6.68, p<.029]$ than following SAL. There were no significant differences between the CLON and SAL conditions on self-ratings of anxiety, affect, and comfort. Following DROP there were changes on all five mood dimensions: subjects felt less alert $[F(1,9)=11.77, p<.008]$, less attentive $[F(1,9)=12.43, p<.006]$, more anxious $[F(1,9)=6.39, p<$ $.032]$, more depressed $[F(1,9)=8.80, p<.016]$, and slightly less comfortable $(p<.081)$ following DROP than following SAL (Table 1).

Behavioral Performance. RT was slower and hit rate lower following CLON than following SAL, but these differences were not significant. DROP led to a significant increase in RT $[F(1,9)=14.39, p<.004)$ and a significant decrease in hit rate $[F(1,9)=18.63, p<.002]$ (Table 1). ${ }^{2}$

Cardiovascular Indices. There were no significant differences between the CLON and SAL conditions in SBP and DBP, but HR was significantly lower $[F(1,9)=6.57$, $p<.03]$ following CLON than following SAL. ${ }^{3}$ There were no differences between the DROP and SAL conditions in SBP, DBP, and HR.

\section{ERP Data}

Grand average ERPs elicited by the four D-stimuli (varying in location and pitch) at $\mathrm{Fz}$ and the $\mathrm{L}+\mathrm{P}+\mathrm{D}+$ target at $\mathrm{Pz}$ for the three drug conditions are shown in Figure 1.

Standards. Mean negativity over 100 to $200 \mathrm{~ms}, 200$ to $400 \mathrm{~ms}$, and 400 to $700 \mathrm{~ms}$ at Fz for the four D-stim-

${ }^{2} \mathrm{RT}$ was more variable following DROP than following SAL or CLON as indicated by analyses of reaction time variance.

${ }^{3}$ The lack of decrease in SBP and DBP following administration of the antihypertensive agent CLON is surprising but is attributable to high preinjection SBP and DBP in the SAL condition (Table 1). 


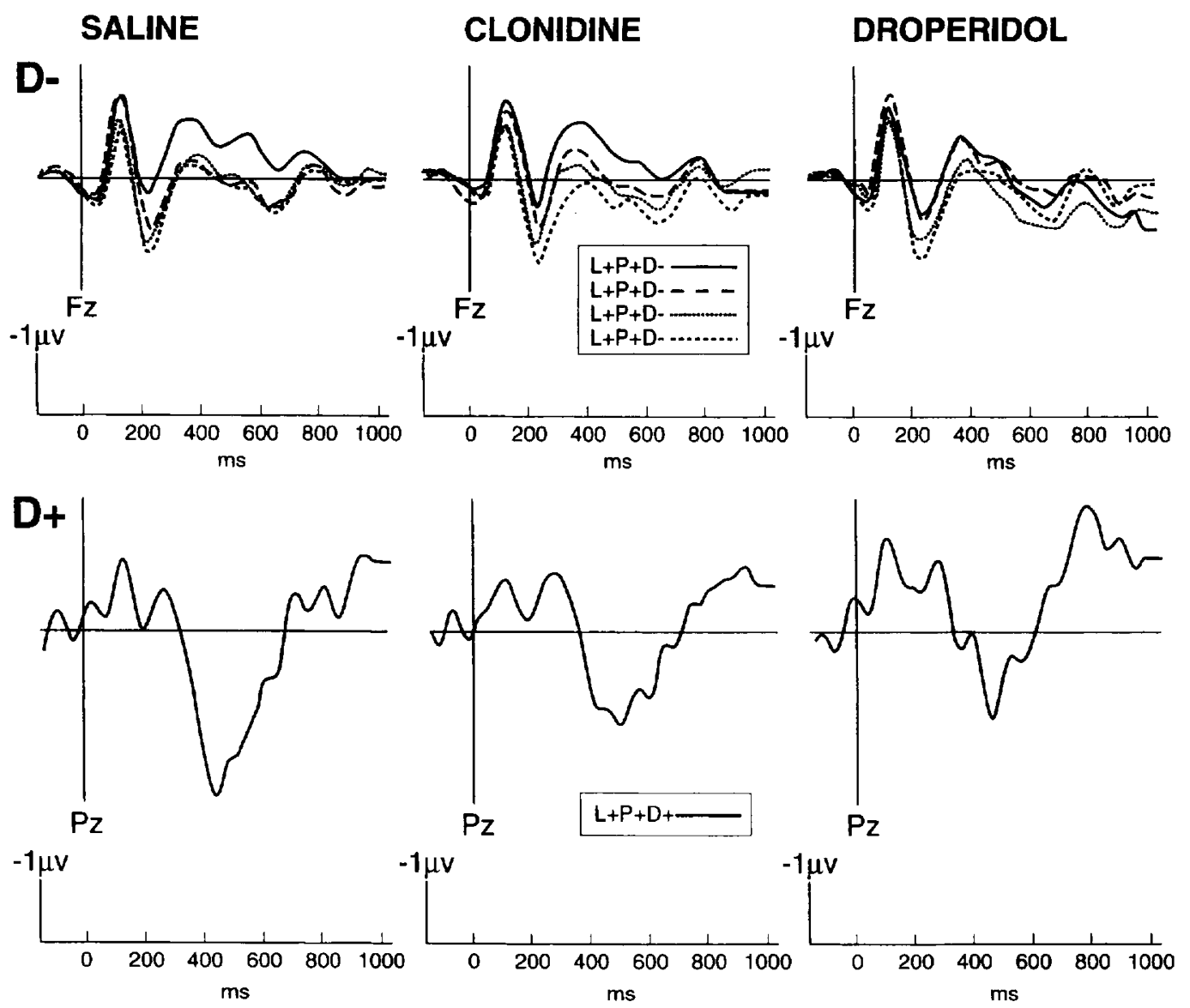

Figure 1. Grand average ERPs following saline, clonidine, and droperidol administration. Top row, ERPs elicited by the $\mathrm{L}+\mathrm{P}+\mathrm{D}-$ (solid lines), $\mathrm{L}+\mathrm{P}-\mathrm{D}-$ (broken lines), $\mathrm{L}-\mathrm{P}+\mathrm{D}-$ (round dots), and $\mathrm{L}-\mathrm{P}-\mathrm{D}-$ (square dots) stimuli at Fz. Note that after 200 $\mathrm{ms}$ in the saline condition pitch processing occurs only at $\mathrm{L}+$ but not at $\mathrm{L}-$; in the clonidine condition there is equal pitch processing at $\mathrm{L}+$ and $\mathrm{L}-$; in the droperidol condition there is no pitch processing at either $\mathrm{L}+$ or $\mathrm{L}-$. Bottom row, ERPs elicited by the $\mathrm{L}+\mathrm{P}+\mathrm{D}+($ solid line) target at $\mathrm{Pz}$.

uli are shown in Table 2. ANOVAs showed that over the 100- to 200-ms epoch drug did not have a significant main effect or drug-by-location interaction either in the CLON versus SAL or in the DROP versus SAL comparisons, indicating that there was early location discrimination under all three conditions. Over the 200to 400-ms epoch, ANOVAs indicated that following CLON there was a significant drug-by-location-bypitch interaction $[F(1,9)=9.66, p<.013]$. This pattern indicates that whereas following SAL, there is pitch processing only at the relevant location $(\mathrm{L}+)$, following CLON, pitch processing occurs both at $\mathrm{L}+$ and at the irrelevant $\mathrm{L}-$. Following DROP there were near-significant interactions between drug by pitch $(p<.057)$ and drug by location by pitch $(p<.066)$ over the 200 - to 400 $\mathrm{ms}$ epoch and a near-significant interaction between drug by pitch $(p<.064)$ over the 400 - to 700-ms epoch. This pattern suggests that following DROP there was a complete disruption of pitch processing and subsequent processes.
Follow-up $t$-tests examined the relative processing of pitch for the SAL, CLON, and DROP conditions separately by comparing the difference between $\mathrm{P}+$ and $\mathrm{P}-$ at the attended and the unattended location over the 200- to 400-ms and 400- to 700-ms epochs. Results are shown in Table 3. Over the 200- to 400-ms epoch a comparison of the difference between $\mathrm{P}+$ and $\mathrm{P}-$ at the attended and the unattended location showed that, whereas following SAL administration, there was significant pitch discrimination at the relevant location $(\mathrm{L}+)(t=5.92, p<.001)$, but not at the irrelevant location $(\mathrm{L}-)(t=.82, p=.433)$, following CLON administration, there was only nearsignificant pitch discrimination at $\mathrm{L}+(t=2.16, p=.059)$, but significant pitch discrimination at $\mathrm{L}-(t=2.76, p=$ .022). Following DROP administration, there was an absence of pitch discrimination at both $\mathrm{L}+(t=.18, p=$ $.863)$ and $\mathrm{L}-(t=.31, p=.766)$. Over the $400-$ to $700-\mathrm{ms}$ epoch, comparisons of pitch discrimination at $\mathrm{L}+$ and $\mathrm{L}-$ indicated that following SAL pitch discrimination continued at $\mathrm{L}+(t=4.05, p=.003)$, but not at $\mathrm{L}-(t=.3$, 
Table 2. ERP Amplitudes Following Clonidine, Droperidol, and Saline Administration ${ }^{a}$

\begin{tabular}{crrr}
\hline & Clonidine & Droperidol & Saline \\
\hline D-stimuli & & & \\
100-200 ms & & & \\
L+P+D- & $-1.08(0.70)$ & $-0.69(0.76)$ & $-1.02(0.71)$ \\
L+P-D- & $-0.84(0.64)$ & $-1.07(0.86)$ & $-0.89(0.77)$ \\
L-P+D- & $-0.49(0.61)$ & $-0.39(0.86)$ & $-0.35(0.78)$ \\
L-P-D- & $-0.20(0.61)$ & $-0.27(0.67)$ & $-0.23(0.57)$ \\
200-400 ms & & & \\
L+P+D- & $-0.31(0.94)$ & $-0.02(0.70)$ & $-0.79(0.63)$ \\
L+P-D- & $0.21(0.73)$ & $0.02(0.52)$ & $0.26(0.46)$ \\
L-P+D- & $0.46(0.67)$ & $0.61(0.92)$ & $0.28(0.69)$ \\
L-P-D- & $1.01(0.66)$ & $0.75(0.83)$ & $0.48(0.48)$ \\
$400-700$ ms & & & \\
L+P+D- & $-1.69(4.30)$ & $-1.42(2.90)$ & $-1.98(2.81)$ \\
L+P-D- & $-0.18(1.59)$ & $-0.01(1.72)$ & $-0.86(1.40)$ \\
L-P+D- & $0.28(1.51)$ & $-0.12(1.63)$ & $-0.47(1.62)$ \\
L-P-D- & $0.25(0.64)$ & $0.34(1.91)$ & $0.13(0.96)$ \\
D+ stimuli & & & \\
P3 mean 300-800 & & & \\
L+P+D- & $1.58(3.76)$ & $0.48(2.53)$ & $1.30(2.55)$ \\
P3 peak amplitude & & & \\
L+P+D+ & $4.40(5.24)$ & $3.86(4.19)$ & $4.87(3.97)$ \\
P3 latency & & & \\
L+P+D+ & $518.5(104.9)$ & $501.1(92.5)$ & $495.5(107.0)$ \\
\hline
\end{tabular}

"SD in parentheses.

"D-stimuli: mean negativity over 100-200ms, $200-400 \mathrm{~ms}$, and $400-700$ $\mathrm{ms}$ at $\mathrm{F} z$.

D+ stimuli: P3 mean amplitude, peak amplitude, and latency over $300-800 \mathrm{~ms}$ at $\mathrm{Pz}$

$p=.773)$. Following CLON there was attenuated pitch discrimination at $\mathrm{L}+(t=2.00, p=.077)$ and no significant pitch discrimination at $\mathrm{L}-(t=1.14, p=.283)$ and following DROP there was no pitch discrimination at either $\mathrm{L}+(t=.34, p=.744)$ or at $\mathrm{L}-(t=.48, p=.644)$.

Targets. Table 2 shows P3 mean amplitude, peak amplitude, and latency for the $\mathrm{L}+\mathrm{P}+\mathrm{D}+\mathrm{ERP}$. Although Figure 1 shows an apparent decrease of $\mathrm{P} 3 \mathrm{am}$ plitude following both drugs, P3 decrements were not

Table 3. T-test Comparisons of the Relative Processing of Pitch at the Attended $(\mathrm{L}+)$ and Unattended $(\mathrm{L}-$ ) Locations over $200-400 \mathrm{~ms}$ and $400-700 \mathrm{~ms}$ at $\mathrm{Fz}$ Following Saline, Clonidine, and Droperidol

\begin{tabular}{lcccccc}
\hline & \multicolumn{2}{c}{ Saline } & \multicolumn{2}{c}{ Clonidine } & \multicolumn{2}{c}{ Droperidol } \\
& $\boldsymbol{t}$ & $\boldsymbol{p}$ & $\boldsymbol{t}$ & $\boldsymbol{p}$ & $\boldsymbol{t}$ & $\boldsymbol{p}$ \\
\hline $\begin{array}{l}200-400 \mathrm{~ms} \\
\mathrm{~L}+\end{array}$ & 5.92 & .001 & 2.16 & .059 & 0.18 & .863 \\
$\mathrm{~L}-$ & 0.82 & .433 & 2.76 & .022 & 0.31 & .766 \\
$400-700 \mathrm{~ms}$ & & & & & & \\
$\mathrm{~L}+$ & 4.05 & .003 & 2.00 & .077 & 0.34 & .744 \\
$\mathrm{~L}-$ & 0.30 & .773 & 1.14 & .283 & 0.48 & .644 \\
\hline
\end{tabular}

statistically significant. (Mean P3 amplitude: CLON vs. SAL $t=.28, p=.787$; DROP vs. SAL $t=.99, p=.35$. peak P3 amplitude: CLON vs. SAL $t=.35, p=.735$; DROP vs. SAL $t=1.18, p=.27$. P3 latency: CLON vs. $\mathrm{SAL} t=.35, p=.735$; DROP vs. SAL $t=.17, p=.869$ ).

\section{DISCUSSION}

Both clonidine and droperidol produced changes in behavioral and ERP indices of selective attention. The present discussion first examines drug-induced changes in behavioral indices, describes the effects of saline, clonidine, and droperidol on ERPs, and finally integrates findings into a framework utilizing the concepts of "tuning" and "switching" (Oades 1985).

The major prediction for the behavioral effects of clonidine was that noradrenergic inhibition would disrupt stimulus processing, reflected in a reduction of hit rate and discrimination, but would have no effect on response processing, reflected in unchanged RTs. A droperidolinduced decrease in chiefly DA was predicted to impair both stimulus processing, as indexed by hit rate and discrimination, and response processing, as indexed by an increase in RT. Consistent with these predictions, clonidine led to a near-significant decrease in hit rate $(p<$ .055) but only a slight, insignificant increase in reaction time. Droperidol led to a significant decrease in hit rate and a significant increase in RT. The droperidol effects are consistent with the results of a previous study that found that it led to a significant decrease in target detection and discrimination and an increase in RT (Clark et al. 1986a). However, clonidine effects are only partly consistent with the results of a previous experiment that found not only decreased target discrimination but also increased RT following clonidine administration (Clark et al. 1986b).

In addition to causing changes in behavioral performance, both substances had significant effects on ratings of mood. After clonidine administration, alertness and attention were reduced, mood effects that are consistent with clonidine's slight impairment of stimulus processing. The effect of droperidol on mood paralleled its adverse effects on behavioral performance, as subjects perceived themselves as less attentive, less alert, more anxious, more depressed, and slightly less comfortable following its administration.

ERPs following saline injection corresponded well to the ERP profile observed in the selective attention task under normal drug-free conditions (Shelley 1991). At about 100 to $200 \mathrm{~ms}$ there was a significant separation of ERPs to standards on the basis of location: "early PN" reflected the separation of ERPs at the attended location $(\mathrm{L}+)$ from ERPs at the unattended location $(\mathrm{L}-)$. At $\mathrm{L}+$, early PN was followed by "later PN" (200-400 ms), reflecting the separation the attended pitch $(\mathrm{L}+\mathrm{P}+)$ from 
the unattended pitch $(\mathrm{L}+\mathrm{P}-)$, but at $\mathrm{L}-$ there was no significant separation of ERPs on the basis of pitch. Thus following saline the more difficult pitch discrimination occurred subsequent to and was contingent on the easy location discrimination, an ERP pattern consistent with models that propose that multidimensional stimuli are normally processed hierarchically (Hansen and Hillyard 1983). Other ERP indices normally observed in the selective attention task also were intact after saline administration: a prolonged "very late PN" in D- ERPs over 400 to $700 \mathrm{~ms}$ at $\mathrm{L}+$ reflected what has been proposed to be the maintenance and rehearsal of an "attentional trace" of the L+P+D- stimulus (Näätänen 1982, 1990). Target stimuli elicited a large parietally maximal P3.

One limitation of the data is that it was not possible to extend the design to include a dose-response manipulation. Future studies should do this. However, the present data still clearly demonstrate the power of ERPs to elucidate pharmacological actions not evident in behavioral performance alone. The most salient effect of clonidine on ERPs was over the D-200- to 400-ms epoch. Comparisons showed that, whereas with saline there was pitch discrimination at $\mathrm{L}+$ but not at $\mathrm{L}-$, with clonidine there was essentially equivalent pitch discrimination of stimuli occurring at both the attended and the unattended locations. The fact that pitch processing continued at $\mathrm{L}-$, the channel that should become irrelevant once the easy dimension (i.e., location) has been found not to match the target, strongly suggests that clonidine led to a disruption of normal hierarchical processing. The pattern suggests that subjects engaged in an alternative strategy involving an independent and exhaustive analysis of stimulus dimensions such as suggested by the models of Deutsch and Deutsch (1963) or Norman (1968). Previous studies on the effects of clonidine on ERPs have found that clonidine led to a reduction in the amplitude of the P3 component elicited by auditory stimuli in both humans (Duncan and Kaye 1986; Joseph and Sitaram 1989) and monkeys (Swick et al. 1988), but did not cause P3 attenuations elicited by visual stimuli in monkeys (Pineda and Swick 1992). In the present study, although grand means suggested a decrease in P3 amplitude with clonidine, the change failed to reach statistical significance.

The disruptive effects of droperidol on ERPs is evident from grand means to standard stimuli (Figure 1). There was no significant difference between the droperidol and saline conditions for the 100- to 200-ms epoch, suggesting that matching for the easy location discrimination was unaffected by droperidol. In contrast, the absence of pitch discrimination was attended as well as the unattended location over the 200- to 400-ms epoch suggested that the processing of the more difficult pitch dimension was disrupted following droperidol. There also were differences between the droperidol and saline conditions over the 400- to 700-ms epoch: whereas fol- lowing saline, processing continued at $\mathrm{L}+$ but not at $\mathrm{L}-$, following droperidol there was an absence of processing at $\mathrm{L}+$ as well as at $\mathrm{L}-$. This suggests that the maintenance and rehearsal processes of the attentional trace of the $\mathrm{L}+\mathrm{P}+\mathrm{D}-$ stimulus that normally occur over this epoch also were impaired by droperidol. The most plausible explanation is that to form an attentional trace of the $\mathrm{L}+\mathrm{P}+\mathrm{D}-$ stimulus, normal location and pitch processing are necessary. With droperidol there was a disruption of pitch processing over the 200- to 400-ms epoch and hence a failure to form an attentional trace that is normally subjected to maintenance and rehearsal processes. Thus the major effect following droperidol administration was a complete breakdown in the processing of the more difficult pitch discrimination and in subsequent processes. Although target ERPs suggested a substantial reduction in P3 amplitude with droperidol (Figure 1), statistically, this did not reach significance.

In terms of Oades' (1985) model of "tuning" and "switching," the main prediction for the effect of clonidine on ERPs was that following decreased noradrenergic transmission, there would be decreased "tuning." Equal pitch processing at both the attended and what is normally the unattended location following clonidine injection was accounted for as the replacement of the usual strategy of hierarchical processing by the far less optimal strategy of independent analysis of stimulus dimensions that involves the unnecessary allocation of attention to the irrelevant location. This also can be viewed as a subject's inability to "tune out" the irrelevant location and a decreased ability to focus on the relevant location, that is, a reduction in tuning.

As droperidol's chief pharmacological effect is a decrease in DA transmission, in terms of the tuning and switching framework, it was predicted that its administration would impair switching from the location to the pitch discrimination, reflected in ERPs as a reduction of pitch PN. Disruptions following droperidol are consistent with an impairment in switching from one relevant stimulus feature (i.e., location) to another (i.e., pitch). These disruptions suggest that subjects are able to perform the easy location discrimination but then find it difficult to switch their attention to the next relevant, and more difficult, stimulus feature of pitch. It should be noted, however, that as well as having effects on attention, droperidol also had significant effects on affect and anxiety and a near-significant effect on comfort. It is therefore possible that some of the observed attentional disruptions are not the direct result of attentional impairments, but were influenced by a range of other factors such as discomfort, malaise, anxiety, and decreased motivation. Subjects simply may not have felt like attending carefully to the task.

The effects of droperidol are also interesting in light of some data on the performance of unmedicated schizophrenic subjects on the SAT. Michie et al. (1990) 
found that, although there was evidence of an early effect of attended location in the standard ERP of schizophrenics, there was a lack of pitch separation to stimuli at the attended location and a total absence of the very late $\mathrm{PN}$ in the $\mathrm{L}+\mathrm{P}+$ standards after $400 \mathrm{~ms}$. Parietal P3s to attended targets also were significantly smaller in schizophrenics. These effects are remarkably similar to some of the effects seen in normal subjects following droperidol administration in the present study. This similarity is paradoxical in view of the fact that droperidol is known to decrease DA transmission, although unmedicated schizophrenics are assumed by most researchers to suffer from a functional overactivity of DA systems. On the basis of these two sets of data it is tempting to speculate that DA modulation of selective attention, and in particular of more difficult discriminations, follows a U-shaped function (Yerkes and Dodson 1908), with either underactivity or overactivity of DA systems leading to impairments.

\section{ACKNOWLEDGMENTS}

This research was supported by a Commonwealth Postgraduate Research Award to Anne-Marie Shelley, the National Health and Medical Research Council, and the Rebecca L. Cooper Medical Research Foundation.

The authors gratefully acknowledge the helpful suggestions and assistance of Daniel C. Javitt, M.D., Ph.D., in the revision and preparation of the final manuscript.

\section{REFERENCES}

Aghajanian GK (1982): Central noradrenergic neurons: A locus for the functional interplay between $\alpha_{-2}$-adrenoceptors and opiate receptors. J Clin Psychiatr 43:20-24

Beninger RJ (1983): The role of dopamine in locomotor activity and learning. Brain Res Rev 6:173-196

Bookbinder J, Osman E (1979): Attentional strategies in dichotic listening. Memory Cog 7:511-520

Clark CR, Geffen GM, Geffen LB (1986a): Role of monoamine pathways in the control of attention: Effects of methylphenidate and droperidol in normal adult humans. Psychopharmacology 90:28-34

Clark CR, Geffen GM, Geffen LB (1986b): Role of monoamine pathways in attention and effort: Effects of clonidine and methylphenidate in normal adult humans. Psychopharmacology 90:35-39

Clark CR, Geffen GM, Geffen LB (1987a): Catecholamines and attention I: Animal and clinical studies. Neurosci Biobehav Rev 11:341-352

Clark CR, Geffen GM, Geffen LB (1987b): Catecholamines and attention II: Pharmacological studies in normal humans. Neurosci Biobehav Rev 11:353-364

Craig CR, Stitzel RE (1982): Modern Pharmacology. Boston, Little, Brown, pp 449-450

Crider A, Solomon PR, McMahon MA (1982): Disruption of selective attention in the rat following chronic d-amphetamine administration: Relationship to schizophrenic attention disorder. Bio Psychiatr 17(3):351-361

Deutsch JA, Deutsch D (1963): Attention: Some theoretical considerations. Psychol Rev 70:80-90

Duncan CC, Kaye WH (1986): Effects of an $a_{2}$-adrenergic agonist on information processing: An electrophysiological analysis. In Rohrbaugh JW, Johnson R, Parasuraman (eds). Eighth International Conference on Event-Related Potentials of the Brain (EPIC VIII): Research Reports, Stanford, CA.

Hansen JC, Hillyard SA (1983): Selective attention to multidimensional auditory stimuli. J Exp Psychol Hum Percep Perform 9(1):1-19

Hawkins HL (1969): Parallel processing in complex visual discrimination. Percep Psychophys 5:56-64

Hornykiewicz O, Kish SJ (1984): Neurochemical basis of dementia in Parkinson's disease. Can J Neurol Sci 11:185-190

Joseph KC, Sitaram N (1989): The effect of clonidine on auditory P300. Psychiatr Res 28:255-262

McCarley RW, Faux SF, Shenton ME, Nestor PG, Adams J (1991): Event-related potentials in schizophrenia: Their biological and clinical correlates and a new model of schizophrenic pathophysiology. Schizophrenia Res 4:209-231

Mason ST (1980): Noradrenaline and selective attention: A review of the model and the evidence. Life Sci 27:617-631

Michie PT, Fox AM, Ward PB, Catts SV, McConaghy N (1990): ERP indices of selective attention and cortical lateralization in schizophrenia. Psychophysiology 27:209-227

Näätänen $R$ (1990): The role of attention in auditory processing as revealed by event-related brain potentials and other brain measures of cognitive function. Brain Behav Sci 13(201):88

Näätänen R (1982): Processing negativity: An evoked-potential reflection of selective attention. Psychol Bull 92:605-640

Näätänen R, Michie PT (1979): Early selective attention effects on the evoked potential: A critical review and reinterpretation. Bio Psychol 8:81-136

Näätänen R, Gaillard AWK, Mantysalo S (1978): Early selective attention effect on evoked potential reinterpreted. Acta Psychologia 42:313-329

Norman DA (1968): Toward a theory of memory and attention. Psychol Rev 75:522-536

Oades RD (1981): Dopaminergic agonistic and antagonistic drugs in the ventral tegmentum of rats inhibit and facilitate changes of food-search behavior. Neurosci Lett $27: 75-80$

Oades RD (1982a): Attention and Schizophrenia: Neurobiological Bases. London, Pitman Advanced Publishing Program

Oades RD (1982b): Search strategies on a hole-board are impaired in rats with ventral tegmental damage: Animal models for tests of thought disorder. Bio Psychiatry $17: 243-258$

Oades RD (1985): The role of noradrenaline in tuning and dopamine in switching between signals in the CNS. Neurosci Biobehav Rev 9:261-282

Peroutka SJ, Snyder SH (1980): Relationship of neuroleptic drug effects at brain dopamine, serotonin, $\alpha$-adrenergic, and histamine receptors to clinical potency. Am J Psychiatr 137:1518-1522 
Pineda JA, Swick D (1992): Visual P3-like potentials in squirrel monkey: Effects of a noradrenergic agonist. Brain Res Bull 28:485-491

Pineda JA, Foote SL, Neville HJ (1989): Effects of locus coeruleus lesions on auditory long-latency event-related potentials in monkey. J Neurosci 9:81-93

Posner MI, Boies SJ (1971): Components of attention. Psychol Rev 78:391-408

Raskin IA, Shatwitz SE, Shaywitz BA, Anderson GM, Cohen DJ (1984): Neurochemical correlates of attention deficit disorder. Ped Clin North Am 31:387-396

Shelley AM (1991): The neural substrate of selective attention: Electrophysiological correlates of catecholamine and endorphin modulation. Unpublished Doctoral Thesis. University of NSW, Sydney, Australia

Shelley AM, Catts SV, Ward PB, Andrews S, McConaghy N (1990): The effects of opioid agonists and antagonists on auditory event related potentials. In Brunia $\mathrm{CH}$, Gaillard AW, et al (eds). Proceedings of EPIC IX, Noordwijck, Holland, vol 2:120-125

Shelley AM, Ward PB, Michie PT, Andrews A, McConaghy
$N$ (1991): The effects of repeated testing on auditory event related potentials. Psychophysiology 28:496-510

Shiffrin RM, Schneider W (1977): Controlled and automatic human information processing: II. Perceptual learning, automatic attending and a general theory. Psychol Rev 84(2):127-190

Snodgrass JG, Townsend JT (1980): Comparing parallel and serial models: Theory and implementation. J Exp Psychol Hum Percep Perform 6:330-354

Svensson T, Bunney B, Aghajanian G (1975): Inhibition of both noradrenergic and serotonergic neurons in brain by the $\alpha$-adrenergic agonist clonidine. Brain Res 92:291-306

Swick D, Pineda JA, Holmes TC, Foote SL (1988): Effects of clonidine on P300-like potentials in squirrel monkeys. Soc Neurosci Abstr 14:1014

Tucker DM, Williamson PA (1984): Asymmetric neural control systems in human self-regulation. Psychol Rev 91(2):185-215

Yerkes RM, Dodson JD (1908): The relationship of strength of stimulus to rapidity of habit formation. J Comp Neurol Psychol 18:459-482 